\title{
Vicker's hardness and Raman spectroscopy evaluation of a dental composite cured by an argon laser and a halogen lamp
}

\author{
Luís Eduardo Silva Soares \\ University of Vale do Paraaíba-UNIVAP \\ School of Dentistry \\ Laboratório de Espectroscopia Vibracional Biomédical \\ Av. Shishima Hifumi, 2911 \\ Urbanova-CEP 12.224-000 \\ São José dos Campos, São Paulo \\ Brazil \\ E-mail: lesoares@univap.br
}

\section{Airton Abrahão Martin}

University of Vale do Paraíba-UNIVAP

Laboratory of Biomedical Vibrational Spectroscopy

Research and Development Institute-IPD

São José dos Campos, São Paulo

Brazil

\section{Antonio Luiz Barbosa Pinheiro \\ University of Vale do Paraíba-UNIVAP \\ Research and Development Institute-IPD \\ São José dos Campos, São Paulo \\ and \\ Federal University of Bahia \\ School of Dentistry \\ Salvador, 40110-150 \\ Brazil}

\section{Marcos T. T. Pacheco}

University of Vale do Paraíba-UNIVAP

Laboratory of Biomedical Vibrational Spectroscopy

Research and Development Institute-IPD

São José dos Campos, São Paulo

Brazil

\begin{abstract}
We present the results of the Vicker's hardness test and the use of near-infrared Raman spectroscopy (RS) to measure in vitro the degree of conversion (DC) of a bis(phenol)-A-glycidyl-dimethacrylatebased composite resin, photoactivated by both a halogen lamp (power density $=478 \mathrm{~mW} / \mathrm{cm}^{2} ; 8-\mathrm{mm}$ diameter spot) and an argon laser (power density $=625 \mathrm{~mW} / \mathrm{cm}^{2} ; 7-\mathrm{mm}$ diameter spot). The degree of conversion was estimated by analyzing the relative intensities between the aromatic $\mathrm{C}=\mathrm{C}$ stretching Raman mode at $1610 \mathrm{~cm}^{-1}$ and the methacrylate $\mathrm{C}=\mathrm{C}$ stretching Raman mode $\left(1640 \mathrm{~cm}^{-1}\right)$ on top and bottom surfaces. For the hardness evaluation, the samples were embedded in polyester resin and three indentations with a 50-g load for $10 \mathrm{~s}$ were made on the top surface. The higher relative DC values achieved by the photoactivation of a composite resin by the argon laser suggest a better biocompatibility in the bottom surface. The correlation test showed that the higher Vicker's hardness number $(\mathrm{VHN})$ values were associated with higher DC values. The derivative analysis showed a greater curing rate from 5 to $20 \mathrm{~s}$ of exposure. The comparison of VHN and DC values with both light sources at each curing time showed that a small change in conversion is related to a large change in hardness. Raman spectroscopy is more sensitive to changes in the first stages of curing reaction than later ones, and the Vicker's hardness assay is more sensitive to changes in the last stages. () 2004 Society of Photo-Optical Instrumentation Engineers. [DOI: 10.1117/1.1688811]
\end{abstract}

Keywords: Vicker's hardness; Raman spectroscopy; degree of conversion; photoactivation; resin restoration.

Paper 02086 received Dec. 9, 2002; revised manuscript received Apr. 23, 2003 and Aug. 21, 2003; accepted for publication Aug. 21, 2003

\section{Introduction}

Although dental composite resins have been widely used as filling materials, they have a major drawback, which is their incomplete polymerization. This can be detected by several methods, including the measurement of the degree of conversion (DC), which is the percentage of double bonds reduced during the polymerization process. Incomplete polymerization results in unreacted monomers, which leach from the material in a wet environment. ${ }^{1}$ Problems associated with inadequate polymerization include poor physical properties, increased solubility in the oral environment (with release of components such as formaldehyde and methacrylic acid), and increased microleakage. All these problems may result in recurrent decay and/or irritation of the pulp.,3

Several studies have been carried out in order to minimize the deficiencies of composite resins influenced by the DC and also to investigate both physical and chemical changes induced by varying the light-curing source. ${ }^{4-11}$ Most of the current photoactivated bis(phenol)-A-glycidyl-dimethacrylate

University of Vale do Paraaíba-UNIVAP, School of Dentistry, Laboratório de Espectroscopia Vibracional Biomédical, Av. Shishima Hifumi, 2911, Urbanova-CEP 12.224-000, São José dos Campos, São Paulo, Brazil. E-mail: lesoares@univap.br
(BIS-GMA)-based composite resins use an initiator such as camphorquinone and a reducing agent such as a tertiary amine to initiate polymerization. This photoinitiator system is sensitive to light in the blue region of the electromagnetic spectrum, with a peak of activity centered around $480 \mathrm{~nm} .^{12}$

Currently a halogen lamp is the most common light-curing unit used by dental clinicians. Since this light source is multiwavelength (400 to $500 \mathrm{~nm}$ ), it is highly absorbed by several types of restorative dental materials. This absorption results in the heating of both the tooth and the resin during the curing process. ${ }^{13}$ Another disadvantage of this curing unit is the limited lifespan of both the bulb and the filter. ${ }^{14,15}$

Recently, attention has turned to the use of an argon laser beam to initiate polymerization of a composite resin. ${ }^{3}$ Previous reports have suggested that polymerization of a composite resin by an argon laser improves its physical properties compared with resins polymerized by a conventional halogen lamp. ${ }^{3}$ One of the main advantages of the argon lasers is its narrow bandwidth, which is centered around $470 \mathrm{~nm}$, which is the optimal wavelength for the activation of the camphorquinone. ${ }^{16}$

1083-3668/2004/\$15.00 @ 2004 SPIE 
Table 1 Technical profiles of the composite resin evaluated.

\begin{tabular}{|c|c|c|c|c|c|c|c|}
\hline Material & Manufacturer & Type & Polymer & Fillers & $\begin{array}{c}\text { Filler } \\
\text { size } \\
(\mu \mathrm{m})\end{array}$ & $\begin{array}{c}\text { Filler } \\
\text { content } \\
\text { (\% by } \\
\text { volume) }\end{array}$ & Shade \\
\hline Z100 & $\begin{array}{l}\text { 3M Dental } \\
\text { Products, St. } \\
\text { Paul, MN. }\end{array}$ & Minifill & $\begin{array}{r}\text { Bis-GMA } \\
\text { TEGDMA }\end{array}$ & $\begin{array}{l}\text { Zirconia } \\
\text { Silica }\end{array}$ & $\begin{array}{c}0.5 \text { to } 0.7 \\
\text { (mean) }\end{array}$ & 66 & A3 \\
\hline
\end{tabular}

Several methods have been reported for studying the DC of composite resins. These include hardness ${ }^{9}$ and optical microscopy ${ }^{10}$ and vibrational methods; i.e., infrared spectroscopy (Fourier-transform infrared) ${ }^{11}$ and Raman spectroscopy (RS). ${ }^{17}$ However, all these studies have used a halogen lamp as a polymerization source.

Vibrational methods allow a precise assessment of the depth of polymerization and the degree of conversion of methacrylate composite resins. ${ }^{5}$ This assessment is based upon the relative intensity of the vibrational bonds of the residual unpolymerized methacrylate $\mathrm{C}=\mathrm{C}$ stretching mode at $1640 \mathrm{~cm}^{-1}$ to the aromatic $\mathrm{C}=\mathrm{C}$ stretching mode at 1610 $\mathrm{cm}^{-1}$, which is used as a standard reference, as has been previously reported. ${ }^{4-6,11,18-21}$ The advantages of Raman spectroscopy over other techniques include little or no sample preparation, the ability to provide direct measurement of unreacted methacrylate groups, and its nondestructive. . $^{17,19,20,22,23}$

Another important method for evaluating the degree of conversion is the hardness assay. ${ }^{3}$ It is known that the DC influences the hardness of composite resins and this depends on several factors, such as time of manufacture or storage, type of polymerization source, color of the resin, polymerization time, postpolymerization time, and intensity of the light and depth of the composite resin. ${ }^{24}$

Although Raman spectroscopy has been used before to evaluate the DC profiles on composite resins cured by a halogen lamp, ${ }^{4-6,11,19,20,25}$ to our best knowledge there are no previous reports on the use of Raman measurements of a composite resin polymerized by an argon laser. Therefore, the aim of this study was to evaluate and correlate Vicker's hardness and the degree of conversion of a BIS-GMA-based composite resin polymerized by both a halogen lamp and an argon laser beam. A comparison of these two methods allowed us to infer the biocompatibility of the resulting filling. This study contributes to a better understanding of the composite resin polymerization process when the resin is cured by an argon laser light. Two methods of photoactivation of composite resin restorative materials were investigated to determine whether they differed significantly in the degree of conversion and hardness value. This information is important to the dental practitioner, not only because the laser is a relatively new tool for curing dental materials, but also because the higher degree of resin polymerization provided by the laser has been associated with improved clinical performance of these materials. $^{12}$

\section{Materials and Methods}

A hybrid proprietary composite resin (Z100, 3M Corp.) was used throughout this study (Table 1). This composite resin is a light-cured radiopaque material. The organic phase (29\%) consists of BIS-GMA, $40 \%$ and triethyleneglycol dimethacrylate (TEGDMA, 60\%). The inorganic material or filler $(71 \%)$ is a mixture of amorphous silica $\left(\mathrm{SiO}_{2}\right)$ and zirconia $\left(\mathrm{ZrO}_{2}\right)$. The filler is not prepared with melted glass or a mineral. It is a synthetic material of zirconia and silica. The filler is ground using new grinding procedures to supply a quite wide distribution of particle sizes, including a high percentage of fine particles. These particles (smaller than $0.1 \mu$ in diameter) supply the handling properties and necessary aesthetics, in this way eliminating the need to increase the amorphous silica. Particle size varies from 3.5 to $0.01 \mu$, with a medium size of $0.6 \mu$. The number of particles per gram is $3.34 \times 10^{15}$. The material is sensitive to visible light, specifically in the region from 400 to $500 \mathrm{~nm}$ of the electromagnetic spectrum. Camphorquinone is the photosensitizer that initiates the photopolymerization of the composite resin.

\subsection{Specimen Preparation}

The composite resin specimens prepared for the Raman measurements were divided into three main groups: an uncured

Table 2 Experimental groups ( $H$, halogen lamp-irradiated samples; $\mathrm{L}$, argon laser-irradiated samples; control, uncured samples).

\begin{tabular}{|c|c|c|c|c|c|}
\hline \multicolumn{3}{|c|}{ Irradiation } & \multicolumn{3}{|c|}{ Irradiation } \\
\hline Groups & $\begin{array}{c}\text { Time } \\
\text { (s) }\end{array}$ & $\begin{array}{c}\text { Number of } \\
\text { Samples }\end{array}$ & Groups & $\begin{array}{c}\text { Time } \\
(\mathrm{s})\end{array}$ & $\begin{array}{l}\text { Number of } \\
\text { Samples }\end{array}$ \\
\hline $\mathrm{H} 5$ & 05 & 05 & L5 & 05 & 05 \\
\hline $\mathrm{H} 10$ & 10 & 05 & L10 & 10 & 05 \\
\hline $\mathrm{H} 2 \mathrm{O}$ & 20 & 05 & L20 & 20 & 05 \\
\hline $\mathrm{H} 30$ & 30 & 05 & L30 & 30 & 05 \\
\hline $\mathrm{H} 40$ & 40 & 05 & L40 & 40 & 05 \\
\hline $\mathrm{H} 60$ & 60 & 05 & L60 & 60 & 05 \\
\hline Control & 0 & 03 & Control & 0 & 03 \\
\hline Total & - & 33 & Total & - & 33 \\
\hline
\end{tabular}




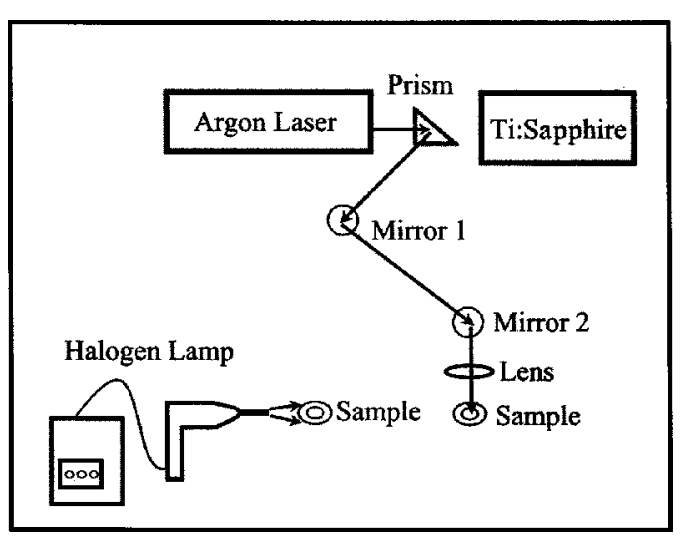

Fig. 1 Schematic of the argon laser and halogen lamp system used to polymerize the samples.

group $(n=6)$ and specimens cured either by a halogen lamp (H group, $n=30$ ) or an argon laser (L group, $n=30$ ) (Table 2). The samples were prepared in a random order. In the cured group, five specimens per exposure were prepared by placing the composite resin in a white circular Teflon mold (7 $\times 2.5 \mathrm{~mm}$ ). The uncured composite paste was compressed and fit to the mold using a condenser and flattened. A Mylar strip (Dentart, POLIDENTAL, São Paulo SP, Brazil; dimension $=10 \times 120 \times 0.05 \mathrm{~mm}$ ) was placed over the top of the mold and pressed flat to extrude the excess composite resin. After insertion of the material into the mold, the specimens were positioned in such a way that the distal end of the curing tip was not in contact with the mold. The samples were then cured either by the halogen light source (Degulux softstart, Degussa-Hüls AG; $\lambda=400$ to $500 \mathrm{~nm}$ ) or the argon laser beam (Stabilite 2017, Spectra Physics; $\lambda=488 \mathrm{~nm}$ ). The exposures for each set of samples were $5,10,20,30,40$, and 60 s. The light sources were applied to the top of the Teflon mold. The power output of the argon laser was kept at 240 $\mathrm{mW}$. The laser beam diameter was focused to $7 \mathrm{~mm}$, the same as the sample diameter, and resulted in a power density of 625 $\mathrm{mW} / \mathrm{cm}^{2}$. The laser beam was diverged by a prism, brought to the sample via two mirrors, and focused by the lens before being delivered to the sample (Fig. 1). The power output of the halogen lamp was $\sim 240 \mathrm{~mW}$, in an 8-mm spot diameter, and the power density was $478 \mathrm{~mW} / \mathrm{cm}^{2}$. Both argon laser and halogen lamp power outputs were measured before the curing process using a power meter (Newport $1835 \mathrm{C}$;) and a curing radiometer (model 100, Demetron Research Corp., Danbury, Connecticut), respectively. After curing, the specimens were removed from the Teflon mold to measure the degree of conversion of both top and bottom surfaces of the specimen using Raman spectroscopy.

\subsection{Raman Spectroscopy}

Raman spectra of both top and bottom surfaces were collected from each sample. Fifty spectra were obtained for each exposure duration and light source, totaling 600 spectra. The Raman signals of the uncured or cured composite resins were acquired by a CCD detector cooled by liquid nitrogen. The samples were excited in the near-infrared region by a titanium:sapphire (Ti:S) laser (model 3900S, Spectra-Physics; $\lambda$ $=830 \mathrm{~nm}$, beam diameter $=1.5 \mathrm{~mm}$ ) pumped by an argon la-

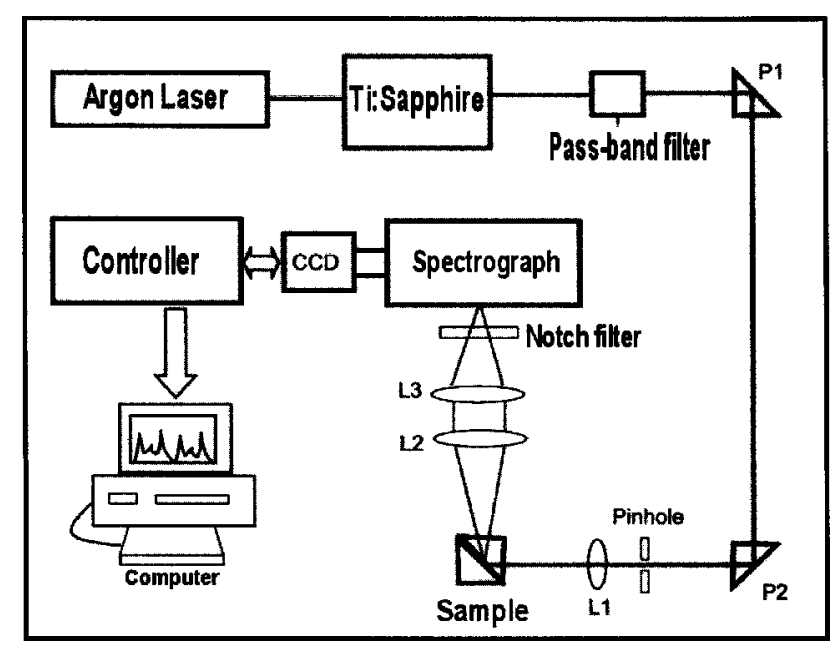

Fig. 2 Near-infrared Raman spectroscopy system used to evaluate the degree of conversion.

ser (Stabilite 2017, Spectra-Physics; $\lambda=488 \mathrm{~nm}$ ) (Fig. 2). The power of the Ti:Sa laser was limited to $50 \mathrm{~mW}$ in the sample holder to avoid additional polymerization of the sample without compromising the sensitivity. ${ }^{20}$ The composite resins of the uncured group were positioned in the sample holder and five spectra were acquired $(n=30)$. Next, the samples cured by different exposures were positioned in the sample holder and five spectra were acquired from five distinct points on both surfaces. WINVIEW software controlled the data acquisition. After data collection, all spectra were processed by LabSpec software to remove the background and to obtain the average spectra. For the uncured samples, five average spectra were obtained $(n=5)$. For the five samples of the group H5 (cured for $5 \mathrm{~s}$ by a halogen lamp), the average of both the five spectra of the top face and the five spectra of the bottom face was calculated. This procedure was repeated for the other samples of the $\mathrm{H}$ group $(\mathrm{H} 10, \mathrm{H} 20$, $\mathrm{H} 30, \mathrm{H} 40, \mathrm{H} 60$ ), thus obtaining 50 more average spectra, and this procedure was also used for each sample of the $\mathrm{L}$ group, obtaining 60 more average spectra $(n=120)$. The Raman vibrational modes at 1640 and $1610 \mathrm{~cm}^{-1}$ of all the average spectra were fitted by a Lorentzian curve. To calculate the degree of conversion of the 120 composite resins from the average spectra, the intensity ratio of the peaks at 1640 and $1610 \mathrm{~cm}^{-1}$ for the cured and uncured resins were used as described earlier. ${ }^{4-6,11,18-21}$ Subsequently, the arithmetic average of the 120 DC values were found and finally 24 DC averages were obtained (Table 3).

\subsection{Vicker's Hardness Test}

The specimens were stored at a relative humidity of 95 $\pm 5 \%$ at $37^{\circ} \mathrm{C}$ in a lightproof container for 1 week. For the Vicker's hardness test, the samples of the twelve irradiation groups (Table 2) were embedded in six blocks of polyester resin (VALGLASS T208, São José dos Campos SP, Brazil), with two groups in each block. The blocks of inclusions were wet polished with a sequence of sandpapers of different grades $(180,400,600)$ to produce flat surfaces. The second polishing was done with diamond paste $(3 \mu \mathrm{m})$ and the final polishing by a colloidal aqueous solution of silica, producing 
Table 3 Mean values of the degree of conversion as a function of irradiation time and polymerization source $(n=5)$.

\begin{tabular}{|c|c|c|c|c|c|c|}
\hline \multirow[b]{2}{*}{ Time (s) } & \multicolumn{3}{|c|}{ DC\% (top) Std. Dev. } & \multicolumn{3}{|c|}{ DC\% (bottom) Std. Dev. } \\
\hline & L & $\mathrm{H}$ & $p$ value & L & $\mathrm{H}$ & $p$ value \\
\hline 5 & $50 \pm 6$ & $36 \pm 3$ & $* * * p<0.001$ & $40 \pm 6$ & $26 \pm 6$ & $* * p<0.01$ \\
\hline 10 & $60 \pm 1$ & $52 \pm 2$ & ns $p>0.05$ & $49 \pm 6$ & $49 \pm 3$ & ns $p>0.05$ \\
\hline 20 & $61 \pm 5$ & $54 \pm 3$ & ns $p>0.05$ & $55 \pm 8$ & $48 \pm 6$ & ns $p>0.05$ \\
\hline 30 & $61 \pm 3$ & $58 \pm 4$ & ns $p>0.05$ & $53 \pm 3$ & $54 \pm 5$ & ns $p>0.05$ \\
\hline 40 & $62 \pm 7$ & $58 \pm 3$ & ns $p>0.05$ & $54 \pm 4$ & $54 \pm 1$ & ns $p>0.05$ \\
\hline 60 & $66 \pm 4$ & $62 \pm 5$ & ns $p>0.05$ & $60 \pm 6$ & $57 \pm 3$ & ns $p>0.05$ \\
\hline
\end{tabular}

a sufficiently flat and parallel surface so that the indentations could be made. Three readings were taken at random positions across the irradiated surface. The indentations were made with a $50 \mathrm{-g}$ load for $10 \mathrm{~s}$ on the top surface of the composite resin using a digital microhardness tester (FM, Future Tech). The mean values were then calculated for each group of light sources and polymerization times.

\subsection{Statistics}

The Raman and Vicker's results were statistically analyzed by two-way analysis of variance (ANOVA) at a 95\% level of confidence. The Tukey-Kramer multiple comparisons post hoc test was also performed using Instat software to test the significance of the degree of conversion and the Vickers hardness number (VHN) in all the exposure times. The post hoc test was done comparing the two light sources and each exposure time. The Kolmorogov and Smirnov tests verified the normal distribution of the sample data. The standard deviations were tested by Barttlet statistics using Instat software. A Pearson $r$ test was carried out to correlate the VHN and the DC values using the Instat software. A derivative analysis was made using Microcal Origin software.

\section{Results}

Figure 3 shows the Raman spectra of the uncured composite resin and for composite resins cured by either a halogen lamp or argon laser irradiation. For better visualization, only the 60-s exposure time is plotted. The Raman spectra show systematic changes in the relative intensities between the peaks at $1610 \mathrm{~cm}^{-1}$ (aromatic $\mathrm{C}=\mathrm{C}$ stretching mode) and at 1640 $\mathrm{cm}^{-1}$ (methacrylate $\mathrm{C}=\mathrm{C}$ stretching mode), as a function of exposure time. As expected, a reduction in the intensity of the peak at $1640 \mathrm{~cm}^{-1}$ was observed on both surfaces with an increase in the exposure time of both light sources [Fig. 3(a) and $3(\mathrm{~b})]$.

The calculated DC of the composite resin (Table 3) showed higher relative values with increasing exposure time on both surfaces, being more linear and homogeneous on ar-
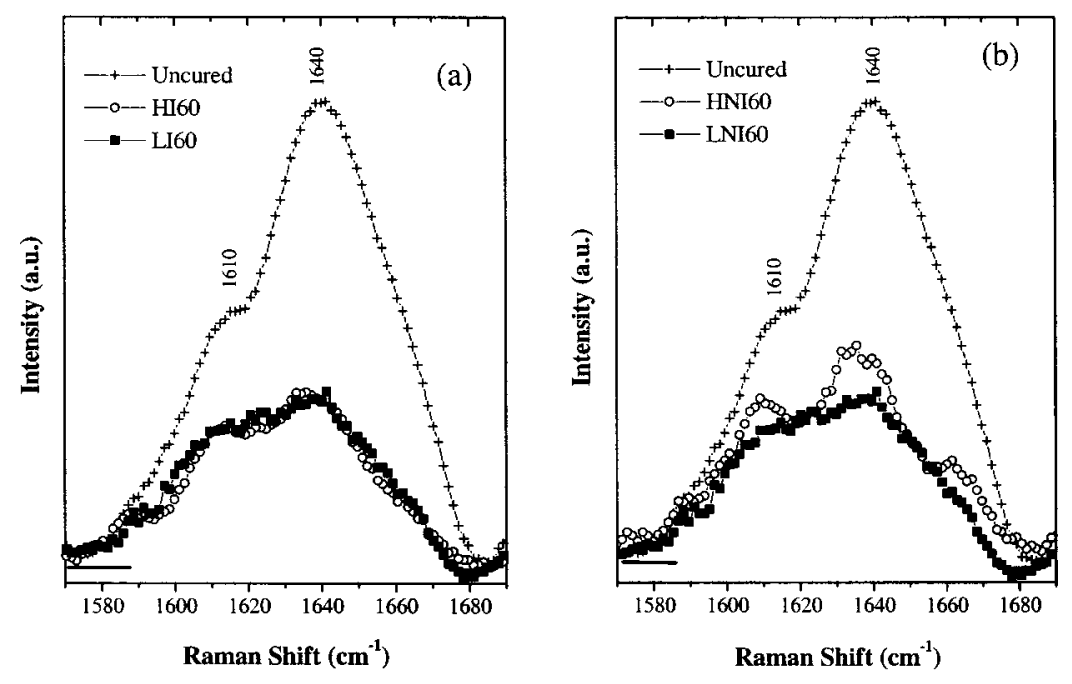

Fig. 3 Raman spectra of composite resin. Uncured group (+) and cured using 60-s exposures under a halogen lamp ( () and an argon laser $(\mathbf{\square})$ on (a) top and (b) bottom surfaces. 
Table 4 Statistical comparison (Tukey-Kramer post hoc test) of the DC in the argon laser and halogen lamp groups.

\begin{tabular}{|c|c|c|c|}
\hline $\begin{array}{c}\text { Comparison } \\
\text { (Top) }\end{array}$ & $p$ value & $\begin{array}{c}\text { Comparison } \\
\text { (Bottom) }\end{array}$ & $p$ value \\
\hline L5 vs. L10 & $* p<0.05$ & L5 vs. L10 & ns $p>0.05$ \\
\hline L10 vs. L20 & ns $p>0.05$ & L10 vs. L20 & ns $p>0.05$ \\
\hline L20 vs. L30 & ns $p>0.05$ & L20 vs. L30 & ns $p>0.05$ \\
\hline L30 vs. L40 & ns $p>0.05$ & L30 vs. L40 & ns $p>0.05$ \\
\hline L40 vs. L60 & ns $p>0.05$ & L40 vs. L60 & ns $p>0.05$ \\
\hline H5 vs. H10 & $* * * p<0.001$ & H5 vs. H10 & $* * * p<0.001$ \\
\hline $\mathrm{H} 10$ vs. $\mathrm{H} 20$ & ns $p>0.05$ & L10 vs. L20 & ns $p>0.05$ \\
\hline $\mathrm{H} 2 \mathrm{O}$ vs. $\mathrm{H} 30$ & ns $p>0.05$ & L20 vs. L30 & ns $p>0.05$ \\
\hline $\mathrm{H} 30$ vs. $\mathrm{H} 40$ & ns $p>0.05$ & L30 vs. L40 & ns $p>0.05$ \\
\hline $\mathrm{H} 40$ vs. $\mathrm{H} 60$ & ns $p>0.05$ & L40 vs. L60 & ns $p>0.05$ \\
\hline
\end{tabular}

gon laser-cured samples. The higher relative DC values for the specimens cured by argon laser and halogen lamp were obtained at $60 \mathrm{~s}$ of exposure time. For the argon laser, the DC reached 66 and $60 \%$ on the top and bottom surfaces, respectively, whereas for the specimens cured by a halogen lamp, the DC values were 62 and $57 \%$ on the top and bottom surfaces, respectively. The Tukey-Kramer multiple comparison post hoc test of DC and VHN values was performed for all times for both light sources and the results are presented in Tables 3, 4, and 5. Comparisons between the DC produced by both light sources showed statistically significant differences for the samples cured by $5 \mathrm{~s}$ on top $(* * * p<0.001)$ and bottom $(* * p<0.01)$ surfaces (Table 3$)$. Analyzing each exposure time (Table 4) for both polymerization sources, statistically significant differences $(* p<0.05)$ were observed for the argon laser samples between the groups with 5 and $10 \mathrm{~s}$ of exposure on the top surface, and for the halogen lamp, extremely significant differences $(* * * p<0.001)$ were observed on the top and bottom surfaces in the same groups. This as-

Table 5 Statistical comparison (Tukey-Kramer post hoc test) of the $\mathrm{VHN}$ in all irradiation groups.

\begin{tabular}{cccc}
\hline $\begin{array}{c}\text { Comparison } \\
\text { Halogen Lamp }\end{array}$ & $p$ value & $\begin{array}{c}\text { Comparison } \\
\text { Argon Laser }\end{array}$ & $p$ value \\
\hline H5 vs. H10 & ns $p>0.05$ & L5 vs. L10 & ns $p>0.05$ \\
H10 vs. H20 & ns $p>0.05$ & L10 vs. L20 & ns $p>0.05$ \\
H20 vs. H30 & ns $p>0.05$ & L20 vs. L30 & ns $p>0.05$ \\
H30 vs. H40 & ns $p>0.05$ & L30 vs. L40 & ns $p>0.05$ \\
H40 vs. H60 & ns $p>0.05$ & L40 vs. L60 & ns $p>0.05$ \\
\hline ns, nonsignificant difference. & &
\end{tabular}

Table 6 Mean values of the Vickers hardness number and standard deviations as a function of irradiation time and polymerization source $(n=5)$.

\begin{tabular}{ccccc}
\hline & \multicolumn{5}{c}{ Vickers Hardness Number (kgf/mm²) } \\
\cline { 2 - 5 } Time (s) & $H$ (Std. Dev.) & $p$ value & $L$ (Std. Dev.) & $p$ value \\
\hline 05 & $125.6 \pm 8.6$ & ns $p>0.05$ & $129.2 \pm 7.0$ & ns $p>0.05$ \\
10 & $133 \pm 3.6$ & ns $p>0.05$ & $143.4 \pm 3.9^{a}$ & ns $p>0.05$ \\
20 & $137 \pm 1.6$ & ns $p>0.05$ & $144.9 \pm 5.2$ & ns $p>0.05$ \\
30 & $137.2 \pm 3.7$ & ns $p>0.05$ & $149.0 \pm 0.5$ & ns $p>0.05$ \\
40 & $147.1 \pm 4.7^{a}$ & ns $p>0.05$ & $156.3 \pm 1.6$ & ns $p>0.05$ \\
60 & $157.7 \pm 6.3$ & ns $p>0.05$ & $161.6 \pm 1.7$ & ns $p>0.05$ \\
\hline \multicolumn{7}{c}{ Mean values with nonsignificant statistical difference. }
\end{tabular}

pect reflects the progressive curing rate, which dropped up to $30 \mathrm{~s}$, and no statistical difference was detectable thereafter.

For the statistical analysis of the Vicker's hardness measurements, the averages of three indentations per sample were calculated, resulting, therefore, in a total of 12 values (Table 6). All irradiation sources tested showed a relative increase in Vicker's hardness values from 5 to $60 \mathrm{~s}$ of exposure time. The Vicker's hardness value reached by the argon laser was 161.6 $\mathrm{kgf} / \mathrm{mm}^{2}( \pm 1.7)$ and for the halogen lamp it was 157.7 $\mathrm{kgf} / \mathrm{mm}^{2}( \pm 6.3)$, both at $60 \mathrm{~s}$ of exposure time. However, statistically the comparisons between the VHN reached by both light sources were nonsignificant $(p>0.05)$ for all exposure times (Table 6). The comparison between each exposure time showed nonsignificant statistical differences $(p>0.05)$ from 5 to $60 \mathrm{~s}$ for specimens cured by both light sources (Table 5).

A correlation test demonstrated exponential behavior between the mean VHN and mean DC values on samples cured by an argon laser [Fig. 4(a)] and a halogen lamp [Fig. 4(b)]. As the hardness increased, a higher DC value was observed. The increase in hardness values showed remarkable changes after the DC reached $\sim 50 \%$ for the halogen light source.

The correlation coefficients found were significantly different from zero. The two-tailed $p$ value for the halogen lamp (0.0453) was considered significant and for the argon laser (0.0044) was considered very significant. The $R^{2}$ values were 0.5868 for group $\mathrm{L}$ and 0.8941 for group $H$. The derivative analysis showed a larger change in the DC of the top surface cured by both light sources from 5 to 20 s of irradiation time; for an irradiation time greater than $20 \mathrm{~s}$, the DC value was kept almost constant [Figs. 5(a) and 5(b)].

The VHN and DC values for argon laser and halogen lamp curing at each irradiation time were compared and the results are shown in Fig. 6 and Tables 3 and 6. A comparison of the DC values for both light sources showed that the argon laser produced a DC of over $60 \%$ with $10 \mathrm{~s}$ of curing time and this remained almost constant until $40 \mathrm{~s}$, when it increased to $66 \%$ at $60 \mathrm{~s}$. For the halogen lamp, the DC is over $60 \%$ only at 60 s of irradiation time [Fig. 6(a)]. A comparison of the VHN values for both light sources showed that the argon laser produced a VHN near $143 \mathrm{kgf} / \mathrm{mm}^{2}$ for 10 to $30 \mathrm{~s}$ of curing time 


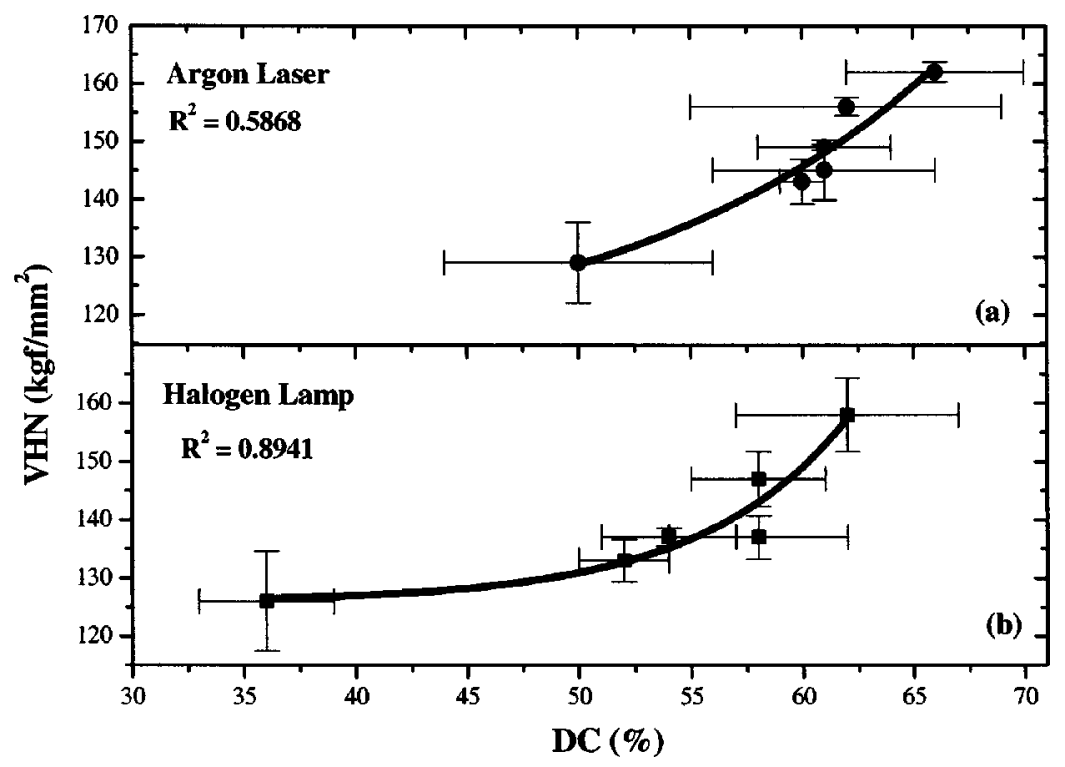

Fig. 4 Correlation between the Vickers hardness number and the degree of conversion for irradiation using (a) an argon laser and (b) a halogen lamp.

and this value increased from 40 to $60 \mathrm{~s}$. For the halogen lamp, a much larger change was noted from 40 to $60 \mathrm{~s}$ of curing time [Fig. 6(b)].

\section{Discussion}

In the present study, a DC evaluation using Raman spectroscopy showed that an argon laser requires $10 \mathrm{~s}$ of curing time to produce an adequate DC $(60 \% \pm 1)$ for the top surface, whereas the halogen light reached this DC value $(62 \% \pm 5)$ only after $60 \mathrm{~s}$ of irradiation of the same surface (Table 2). The major statistical difference observed in the DC for the samples cured by argon laser for 5 and $10 \mathrm{~s}$ on the top surface, is probably due to the initially high curing rate produced by the laser, which drops with increasing time. For the halogen lamp group, the major statistical difference in this same irradiation time on both surfaces is probably due to the broader range of wavelengths of this conventional light source, since only a small portion of this band is efficient in the curing process.

For the bottom surface, the argon laser resulted in a reduction of the curing time and in a greater reduction in carbon double bonds. The DC value reached by argon laser irradiation at deep portions of the composite resin indicates the presence of less residual toxic monomer and, consequently, im-

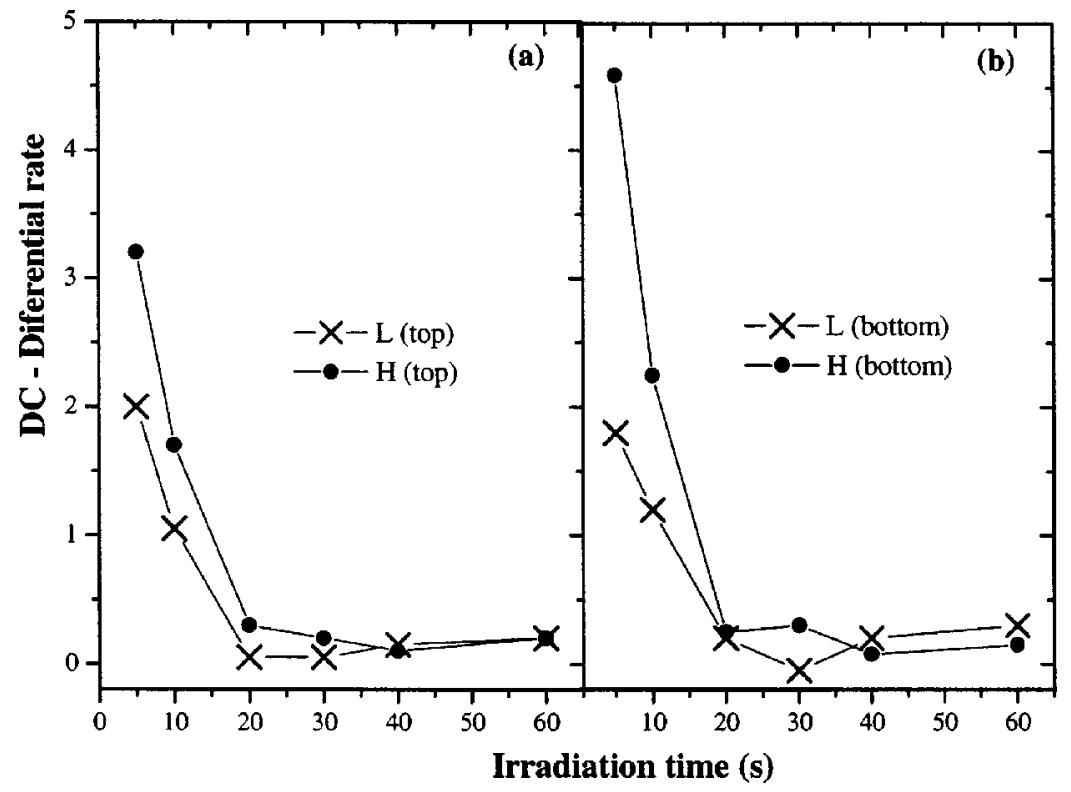

Fig. 5 Derivative analysis of all irradiation times for the DC values (Table 3) produced by irradiation by the argon laser (X) and halogen lamp at (a) top and (b) bottom surfaces. 


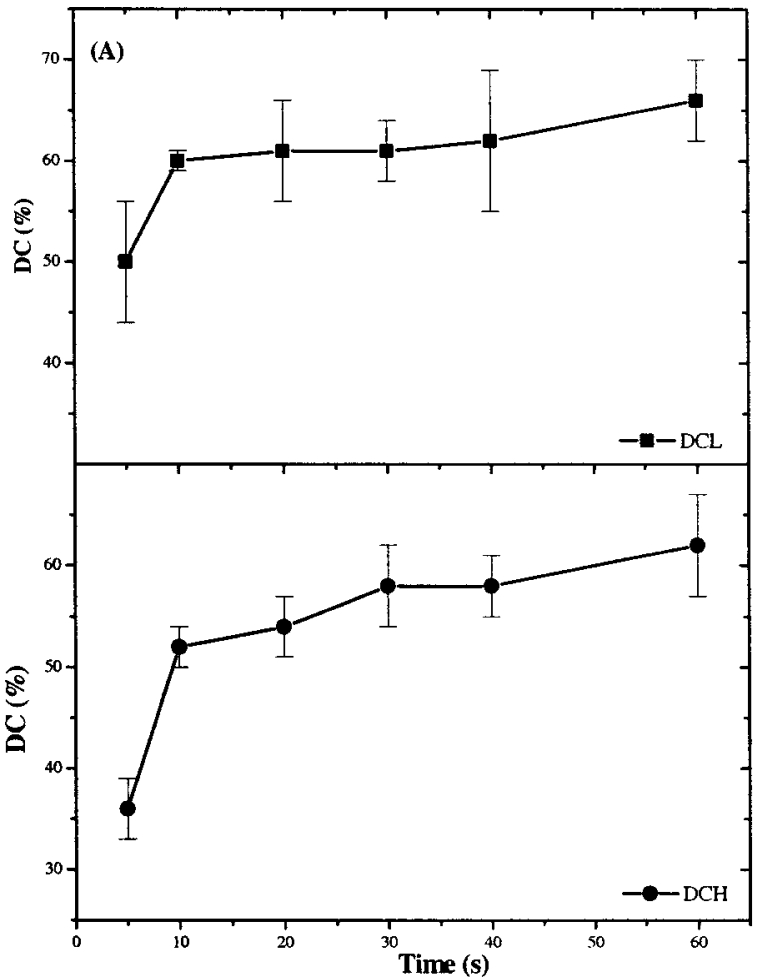

Fig. 6 Comparison of (a) DC and (b) VHN values between argon laser

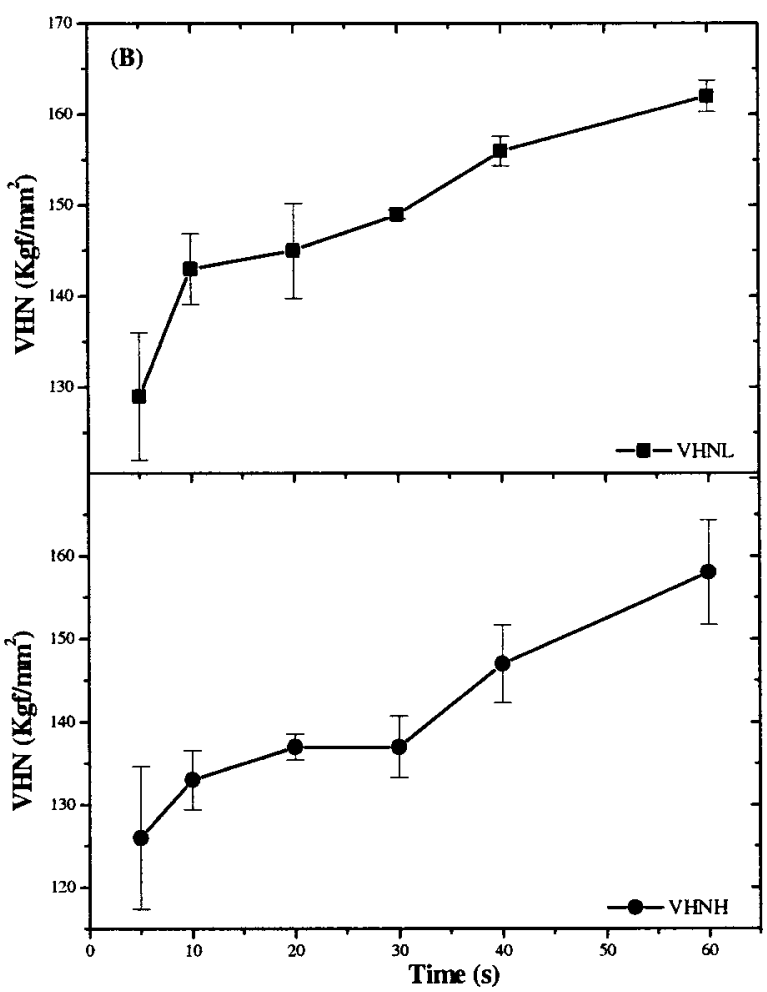

and halogen lamp (-) curing at each curing time. proved biocompatibility of the filling. ${ }^{1-3}$ However, for the halogen lamp, a similar DC value was not reached, showing that for the bottom surface, at a depth of $2.5 \mathrm{~mm}$, more residual monomer was present.

The observed differences in DC for top and bottom are probably due to the thickness of the samples. The thicker the layer is, the more difficult it is for the light to reach deeper portions of the composite resin to activate the polymerization reaction. Consequently, there will be a smaller degree of conversion and therefore a reduction in the hardness. Another aspect to consider is that the greater penetration depth of the argon laser allows the curing of larger increments of composite resin or curing through thicker sections of tooth, producing greater DC values. It could also make it possible to control the direction of polymerization shrinkage by curing through the tooth. $^{22}$

In the current study, an increase in mean relative hardness values associated with an increased exposure time for both light sources was observed. The degree of polymerization in the argon laser-cured samples indirectly observed by a correlation with the VHN was greater with a reduced exposure time than that obtained when the halogen lamp was used, confirming the Raman spectroscopy data. This is probably due to the higher energy density values produced by the laser (i.e., it delivers more total energy than the halogen lamp ${ }^{3}$ ) and also to laser light characteristics, such as coherence, collimation, and monochromaticity. ${ }^{3}$ In addition to energy density, the wavelength of the emitted light should be considered in determining a light source's efficiency for polymerization of a composite resin. ${ }^{3}$ Although conventional light sources emit a broader range of wavelengths (400 and $520 \mathrm{~nm}$ ), only a small portion of this band is efficient in polymerizing a composite resin. ${ }^{3}$ In contrast the argon laser peak emission is centered at $488 \mathrm{~nm}$, which is close to the absorption peak of camphorquinone and therefore is more effective in curing composite resins and produces an increased hardness.

The correlation between mean VHN and DC values shows that higher VHN values indicate higher DC values, confirming the indirect measurement of the DC of the composite resin by VHN and the direct measurement of the DC by Raman spectroscopy. This result showed that with increasing conversion values, the slope of the prediction line increases (Fig. 4) and consequently a small change in conversion will be related to a large change in hardness. This means that as hardness increases, the ability to distinguish between conversion degree increases, confirming the results of an earlier study. ${ }^{11}$ The derivative analysis in Fig. 5 indicates the higher curing capability of these two sources with 5 to $20 \mathrm{~s}$ of exposure.

A comparison of DC with the argon laser and halogen curing units (Fig. 6) at each time shows that the argon laser produces a DC over $60 \%$ and is much faster than the halogen lamp. However, the VHN comparison showed a larger change in the hardness values for each curing time. Thus it can be seen that a small change in conversion degree will be related to a large change in hardness, showing that Raman spectroscopy is sensitive to larger changes in the DC in the first stages of a curing reaction, whereas with the Vicker's hardness assay, this occurs in the last stages of the reaction.

As dental technology continues to advance, new methods for performing some dental procedures will continue to replace the old ones, the use of the argon laser to cure composite resins being an example. The use of the laser to cure composite resins clearly demonstrates a greater degree of polymerization than that achieved by a halogen lamp. This 
enhanced polymerization improves physical properties and bond strengths, ${ }^{22}$ and consequently has important clinical implications for preventing early failures of restorations. When the composite resin is cured by an argon laser, better polymerization occurs, which is also important in reducing adverse pulpal sensitivity. ${ }^{22}$ The reduction in polymerization times provided by the argon laser reduces treatment time. In the present investigation, the argon laser produced better results for both the DC and the superficial hardness of the composite resin than a halogen lamp. However, the main disadvantage of the use of the argon laser is its high cost and higher shrinkage values. This should stimulate the development of new cheaper and more efficient light-curing sources.

\section{Conclusion}

This study showed that Raman spectroscopy could be used to assess the progression of the conversion of composite resins. The higher DC values for a composite resin obtained by polymerization using an argon laser suggest a better biocompatibility on the bottom surface $(2.5 \mathrm{~mm})$. A correlation test showed that higher VHN values were associated with higher DC values. A derivative analysis showed a larger curing rate for 5 to $20 \mathrm{~s}$ of exposure. A comparison of $\mathrm{VHN}$ and DC values for both light sources at each curing time showed that a small change in conversion is related to a large change in hardness. Raman spectroscopy is more sensitive to changes in the first stages of curing reaction than later ones and the Vicker's hardness assay is more sensitive to changes in the last stages.

\section{Acknowledgments}

We thank Steven Frederic Durrant for a critical reading of the manuscript. This work was supported by IP\&D-UniVap and CNPq grant no. 302393/2003-0.

\section{References}

1. A. U. J. Yap, H. K. Lee, and R. Sabapathy, "Release of methacrylic acid from dental composite," Dent. Mater. 16, 172-179 (2000).

2. A. Peutzfeldt, "Resin composites in dentistry: the monomer systems," Eur. J. Oral Sci. 105, 97-116 (1997).

3. M. A. Vargas, D. S. Cobb, and J. L. Schmit, "Polymerization of composite resins: argon laser vs. conventional light," Oper. Dent. 23, 87-93 (1998)

4. J. Xu, I. S. Butler, D. F. R. Gibson, and I. Stangel, "High-pressure infrared and FT-Raman investigation of a dental composite," Biomaterials 18(24), 1653-1657 (1997).

5. C. Pianelli, J. Devaux, S. Bebelman, and G. Leloup, "The microRaman spectroscopy, a useful tool to determine the degree of conversion of light-activated composite resins," J. Biomed. Mater. Res. 48, 675-681 (1999).

6. S. A. Lundin and G. Koch, "Cure profiles of visible-light-cured class II composite restorations in vivo and in vitro," Dent. Mater. 8, 7-9 (1992).
7. K. J. Anusavice, "Phillips science of dental materials," in Restorative Resins, 10th ed., pp. 273-300, W. B. Saunders, Philadelphia, PA (1996).

8. A. Meniga, Z. Tarle, M. Ristic, J. Sutalo, and G. Pichler, "Pulsed blue laser curing of hybrid composite resins," Biomaterials 18(20), 1349-1354 (1997)

9. H. Matsumoto, J. E. Gres, V. A. Marker, T. Okabe, J. L. Ferracane, and G. A. Harvey, "Depth of cure of visible light-cured resin: clinical simulation," J. Prosthet. Dent. 2, 80-84 (1986).

10. S. M. Newman, G. A. Murray, and J. L. Yates, "Visible lights and visible light-activated composite resins," J. Prosthet. Dent. 50, 31-35 (1983)

11. A. Rueggeberg, D. T. Hashinger, and C. W. Fairhust, "Calibration of FTIR conversion analysis of contemporary dental resin composites," Dent. Mater. 6, 241-249 (1990).

12. R. J. Blankenau, W. P. Kelsey, G. L. Powell, G. O. Shearer, W. W. Barkemeier, and W. T. Cavel, "Degree of composite resin polymerization with visible light and argon laser," Am. J. Dent. 4, 40-42 (1991).

13. C. Kurachi, A. M. Tuboy, D. V. Magalhães, and V. S. Bagnato, "Hardness evaluation of a dental composite polymerized with experimental LED-based devices," Dent. Mater. 17, 309-315 (2001).

14. M. Miyazaky, T. Hattori, Y. Ichiishi, M. Kondo, H. Onose, and B. K. Moore, "Evaluation of curing units used in private dental offices," Oper. Dent. 23, 50-54 (1998).

15. F. E. Martin et al., "A survey of the efficiency of visible light curing units," J. Dent. 26, 239-243 (1998).

16. W. P. Kelsey, R. J. Blankenau, G. L. Powell, W. W. Barkmeier, and E. F. Stormberg, "Power and time requirements for use of the argon laser to polymerize composite resins," J. Clin. Lasers Med. Surg. 10, 273-278 (1992).

17. J. F. Aust, K. S. Booksh, C. M. Stellman, R. S. Parnas, and M. L. Myrick, "In situ analysis of a high-temperature cure reaction in real time using modulated fiber-optic FT-Raman spectroscopy," Appl. Spectrosc. 53(6), 682-686 (1999).

18. L. E. S. Soares, A. A. Martin, and A. L. B. Pinheiro, "Raman study of composite resins polymerized by halogen lamp and argon laser," Proc. SPIE 4610, 15-20 (2002).

19. J. W. Stansbury and S. H. Dickens, "Determination of double bond conversion in dental resins by near infrared spectroscopy," Dent. Mater. 17, 71-79 (2001).

20. W. S. Shin, X. F. Li, B. Schwartz, S. L. Wunder, and G. R. Baran, "Determination of the degree of cure of dental resins using Raman and FT-Raman spectroscopy," Dent. Mater. 9, 317-124 (1993).

21. J. D. Louden and T. A. Roberts, "Cure profiles of light-cured dental composites by Raman spectroscopy," J. Raman Spectrosc. 14(5), 365-366 (1983).

22. M. G. Fleming and W. A. Maillet, "Photopolymerization of composite resin using the argon laser," J. Can. Dent. Assoc. 65, 447-450 (1999).

23. B. Van Meerbeek, H. Morbacher, J. P. Celis, J. R. Roos, M. Braem, P. Lambrechts, and G. Vanherle, "Chemical characterization of the resin-dentin interface by micro-Raman spectroscopy," J. Dent. Res. 72(10), 1423-1428 (1993).

24. L. A. Santos, M. L. Turbino, M. N. Youssef, and E. Matson, "Microhardness of composite resins: effect of photocuring units and polymerization periods in different depths," Pesquisa Odontológica Brasileira 14(1), 65-70 (2000).

25. M. Miyazaki, H. Onose, N. Iida, and H. Kazama, "Determination of residual double bonds in resin-dentin interface by Raman spectroscopy,” Dent. Mater. 19, 245-251 (2003). 